\title{
MIELOMA MÚLTIPLE: CLÍNICA, PARACLÍNICA Y PRINCIPALES ESQUEMAS DE TRATAMIENTO HOSPITAL DE SAN JOSÉ DE BOGOTÁ DC. 2003 A 2010
}

\author{
Virginia Abello Polo MD*, Jair Figueroa Emiliani MD**, Licet Villamizar***
}

\section{Resumen}

El mieloma múltiple es una neoplasia de células plasmáticas incurable, con morbilidad discapacitante por la gran sintomatología y una alta mortalidad. Afecta todas las razas y áreas geográficas, y su edad media de diagnóstico es de 66 años con solo $10 \%$ menores de 50 años y $2 \%$ por debajo de 40 . Objetivo: describir las características clínicas, paraclínicas y los principales esquemas de tratamiento en primera línea de los pacientes manejados y tratados en el Hospital de San José de Bogotá DC, entre enero 2003 y diciembre 2010. Método: cohorte retrospectiva de mayores de 18 años de edad con diagnóstico de mieloma múltiple sintomático. Los datos fueron extraídos de las historias clínicas. Resultados: 39 pacientes se diagnosticaron durante ocho años, $59 \%$ hombres cuya edad promedio fue de 59,7 años (DE de 10,7). El 70\% fue considerado candidato a trasplante y en el $33,0 \%(n=7 / 21)$ de estos se efectuó el procedimiento. El esquema de quimioterapia más usado fue talidomida-dexametasona (23\%); la respuesta al tratamiento más común fue muy buena parcial $(\mathbf{4 0 , 9 \%})$ y el $23,7 \%$ alcanzaron por lo menos una completa.

Palabras clave: mieloma múltiple, tratamiento, trasplante autólogo de células madres hematopoyéticas, neoplasia de células plasmáticas, recaída.

\section{MULTIPLE MYELOMA: CLINICAL AND LABORATORY MANIFESTATIONS AND MAIN TREATMENT REGIMENS HOSPITAL DE SAN JOSÉ DE BOGOTÁ DC. 2003 TO 2010}

\begin{abstract}
Multiple myeloma is an incurable cancer of the plasma cells featuring disabling morbidity due to its many possible symptoms and high mortality rate. It affects all races and geographic regions. The average age at diagnosis is 66 years. Only $10 \%$ are under age 50 and $2 \%$ under $\mathbf{4 0}$. Objective: to describe the clinical and laboratory manifestations and main first-line therapies of patients managed and treated at Hospital de San José, Bogotá DC, between January 2003 and December 2010. Method: a retrospective cohort study of patients with diagnosed symptomatic multiple myeloma aged more than 18 years based on data compiled from clinical records. Results: thirty-nine (39) patients were diagnosed in an 8-year period, $59 \%$ were men with a mean age of 59.7 years (SD 10.7). Seventy percent $(70 \%)$ were considered candidates for a transplant and this procedure was actually performed in $33.0 \%(n=7 / 21)$. The chemotherapy regimen most commonly used was a thalidomide-dexamethasone combination (23\%). The most frequently observed response to therapy was a very good partial response $\mathbf{( 4 0 . 9 \% )}$ ) and $23.7 \%$ achieved at least one complete response.
\end{abstract}

Key words: multiple myeloma, treatment, autologous hematopoietic stem cell transplantation, cancer of the plasma cells, relapse.

Fecha recibido: diciembre 9 de 2011 - Fecha aceptado: enero 27 de 2012

* Profesora Asistente, Fundación Universitaria de Ciencias de la Salud. Servicio de Hematología, Hospital de San José, Bogotá DC. Colombia.

\footnotetext{
* Residente II de Hematología, Fundación Universitaria de Ciencias de la Salud. Bogotá DC. Colombia.

*** Profesora Asistente, División de Investigaciones, Fundación Universitaria de Ciencias de la Salud. Bogotá DC. Colombia.
} 


\section{Introducción}

El mieloma múltiple es una neoplasia linfoide de célula B madura que ocasiona cerca del $20 \%$ de las muertes por neoplasias malignas hematológicas y $2 \%$ de todos los cánceres. ${ }^{1}$ Es una enfermedad incurable, propia de adultos mayores de todas las razas y áreas geográficas, con una edad media de diagnóstico de 66 años. ${ }^{2,3} \mathrm{Su}$ presencia cursa con una morbilidad discapacitante por la gran sintomatología así como una alta mortalidad. ${ }^{1}$

Representa alrededor del $1 \%$ de todos los cánceres y un poco más del 10\% de las neoplasias hematológicas en los Estados Unidos, con una incidencia anual de 4 a 5 por 100.000 habitantes. ${ }^{4}$ Para 2008 según Globocan se reportaron 102.000 nuevos casos a nivel mundial, con una incidencia similar para ambos sexos. ${ }^{5}$ En el Instituto Nacional de Cancerología de Bogotá DC, Colombia, para el año 2009 fue de 24 casos. $^{6}$

\section{La Mayo Clinic y The International Myeloma Working} Group para diagnóstico de mieloma múltiple sintomático, enfatizan la importancia del daño de órgano blanco para establecer el diagnóstico..$^{7-9}$ Los tres criterios necesarios para el mieloma múltiple sintomático incluyen presencia de componente monoclonal en suero y/u orina, infiltración de $10 \%$ o más de células plasmáticas neoplásicas en médula ósea y evidencia de lesión de órgano o de tejido atribuida a mieloma conocido como componente CRAB (increased plasma calcium level, renal insufficiency, anemia, and lytic bone lesions). ${ }^{9}$ El pronóstico de esta entidad se determina según el International Staging System (ISS) de acuerdo con los niveles séricos de albúmina y de B2 microglobulina ${ }^{9,10}$ y por las anormalidades cromosómicas identificadas por cariotipo convencional y/o hibridación in situ por fluorescencia (FISH) ${ }^{9.11}$ Luego del diagnóstico, la mediana de supervivencia sin tratamiento efectivo es de cerca de seis meses ${ }^{12}$; después de terapia convencional de tres a cuatro años y con altas dosis de quimioterapia seguidas de trasplante autólogo de células madre hematopoyéticas puede prolongarla a cinco o siete años. ${ }^{12,13}$

El tratamiento inicial ante mieloma sintomático varía dependiendo de si son candidatos o no a trasplan- te autólogo de células madre hematopoyéticas ${ }^{9,14-16}$, que tiene un tasa de supervivencia global y libre de enfermedad muy superior a la evidenciada con quimioterapia combinada, de tal forma que todos los que sean diagnosticados con mieloma múltiple deben ser evaluados para definir la posibilidad de ser llevados a dicho procedimiento. ${ }^{15,17}$

El Hospital de San José de Bogotá DC, (Colombia) es un centro de referencia nacional para el manejo de patologías neoplásicas hematológicas con una amplia experiencia en mieloma múltiple, que cuenta con adecuadas herramientas diagnósticas y una buena infraestructura para la administración de quimioterapia de inducción. A lo largo de los años se ha utilizado una gran variedad de esquemas quimioterapéuticos solos o combinados con trasplante autólogo de células madre hematopoyéticas. Sin embargo, no contamos con datos propios actualizados sobre las características clínicas y paraclínicas, tasas de respuesta de los diferentes esquemas de tratamiento y porcentaje de pacientes llevados a trasplante.

La motivación para la realización de este trabajo se deriva de la necesidad de conocer en detalle estas características en nuestros pacientes durante los últimos siete años y comparar nuestros resultados con los reportados en la literatura internacional y, de manera consecuente, realizar los ajustes necesarios en el fortalecimiento y mantenimiento de un adecuado nivel desde el punto de vista diagnóstico y terapéutico en relación con esta patología, que nos permita seguir siendo centro de referencia nacional.

\section{Métodos}

El trabajo se desarrolló de manera retrospectiva mediante la revisión de las historias clínicas de los pacientes atendidos en las áreas de consulta externa y hospitalización del servicio de Hematología del Hospital de San José de Bogotá DC, Colombia, entre los años 2003 y 2010, obteniendo información sobre las características clínicas y paraclínicas de cada uno ellos, así como de los diferentes esquemas terapéuticos usados en primera línea y sus tasas de respuesta. 
Ingresaron todos los pacientes mayores de 18 años de edad que cumplían los criterios diagnósticos para mieloma múltiple sintomático según el International Myeloma Working Group, que recibieron tratamiento de primera línea en el Hospital de San José entre enero 2003 y diciembre 2010. Los datos se recolectaron en un formato diseñado por el investigador. Con el fin de establecer la proporción de pacientes vivos durante la realización del estudio, se contactó por vía telefónica a los enfermos o familiares y se consultó la página del Ministerio de la Protección Social de la República de Colombia, SISPRO (Sistema Integral de Información de la Protección Social) explorando el registro único de afiliados (RUAF).

Las variables numéricas se describen por medio de medidas de tendencia central y de dispersión. Las variables categóricas se presentan como proporciones. Los datos se digitaron en una base de datos construida en Excel y la información se analizó en el programa Stata versión 10.0.

\section{Resultados}

La frecuencia anual de pacientes nuevos con mieloma múltiple sintomático en el Hospital de San José en los últimos ocho años se expresa en la Figura 1. Ingresaron 39 con mieloma múltiple sintomático de los cuales 53,8\% $(n=21)$ fueron menores de 60 años de edad al momento del diagnóstico y $59 \%$ de género masculino, la edad promedio fue 59,7 años (DE 10,7). El 53,8\% (n=21) de ellos menores de 60 años y 20,5\% $(n=8)$ inferior a 50.

Las principales manifestaciones clínicas y paraclínicas se muestran en la Tabla 1. Las más frecuentes fueron dolores óseos y síntomas B $(71,7 \%$ y $61,6 \%)$ y los hallazgos documentan un gran porcentaje con niveles de 32 microglobulina mayor de $3.5 \mathrm{mg} / \mathrm{dl}(55 \%)$ y con más de tres lesiones líticas $(46,1 \%)$. En la Tabla 2 se detalla la estadificación de la enfermedad según los dos principales sistemas usados para tal fin (Durie-Salmon e ISS) poniendo de manifiesto que la mayoría presentaban un estadio avanzado de enfermedad (Durie-Salmon estadio III: $89,7 \%$ ) y un riesgo intermedio o alto $(68,8 \%)$.

Al evaluar el reporte del componente $\mathrm{M}$ en las historias clínicas se encontró lo siguiente:

- Mediante electroforesis de proteínas se evidenció pico monoclonal en $78,1 \%$ de 32 que presentaban

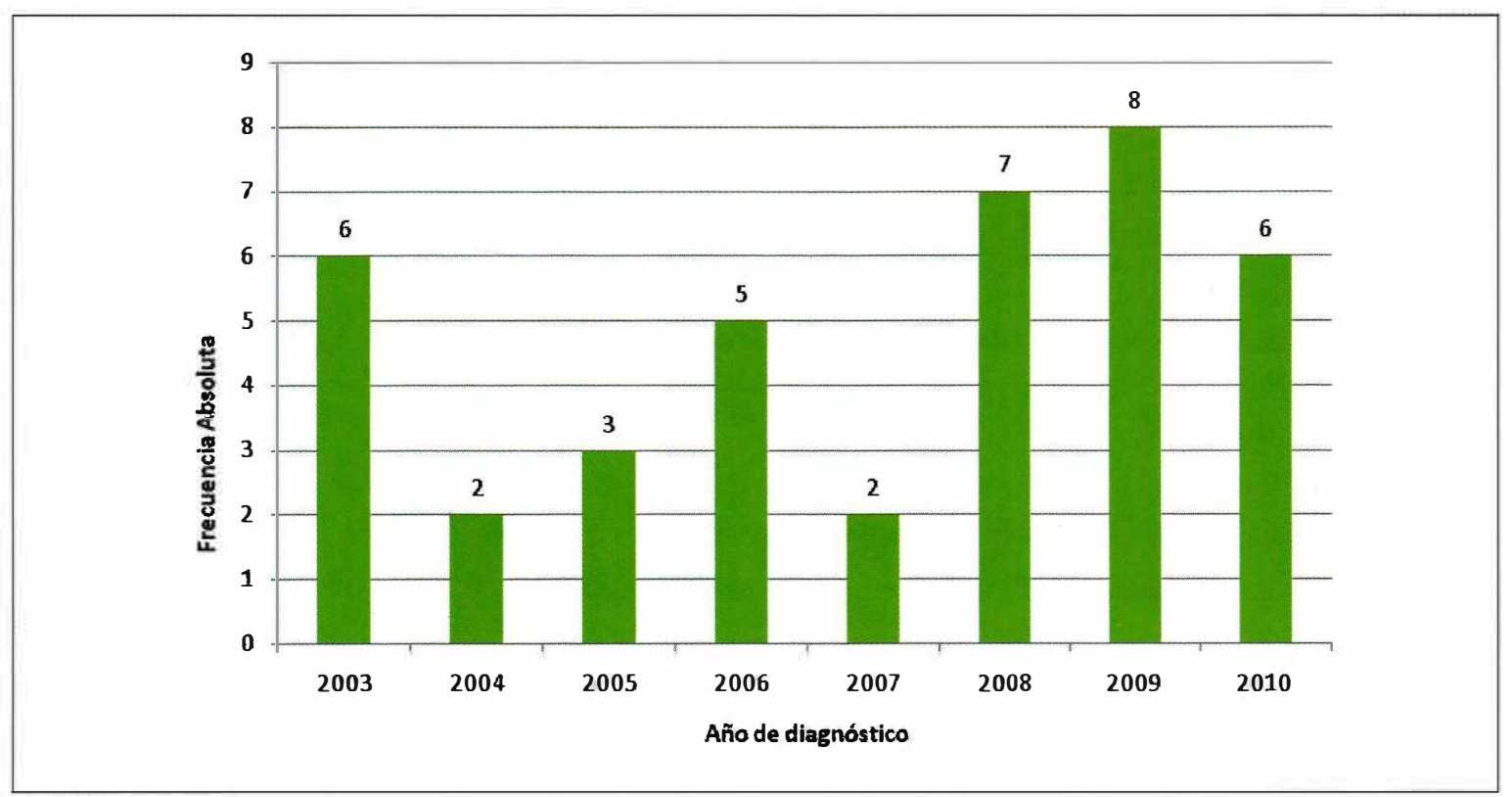

Figura I.Frecuencia anual de pacientes con diagnóstico nuevo de mieloma múltiple sintomático en el Hospital de San José, 2003-20I0. 


\begin{tabular}{|c|c|c|}
\hline \multicolumn{2}{|c|}{ Caracteristicas clínicas } & (\%) n \\
\hline \multirow{3}{*}{ Manifestaciones esqueléticas } & Dolores óseos & $71.7(28)$ \\
\hline & Fracturas patológicas & $35.9(14)$ \\
\hline & Plasmocitoma vertebral & $2.5(1)$ \\
\hline \multirow{3}{*}{ Síntomas sistémicos } & Sintomas B & $61.5(24)$ \\
\hline & Astenia, adinamia, malestar general & $43.5(17)$ \\
\hline & Hipercalcemia sintomática & $15.3(6)$ \\
\hline \multirow{2}{*}{ Infecciones a repetición } & Respiratoria & $2.5(1)$ \\
\hline & Absceso glúteo & $2.5(1)$ \\
\hline \multirow{3}{*}{ Síntomas neurológicos } & Parestesias & $5.1(2)$ \\
\hline & Signos de compresión radicular & $5.13(2)$ \\
\hline & Alteración de conciencia & $5.13(2)$ \\
\hline Caracteristicas & $\begin{array}{c}\text { Caracteristicas paraclínicas } \\
\text { Concentración }\end{array}$ & (\%) $n$ \\
\hline \multirow{3}{*}{$\begin{array}{l}\text { Hemoglobina sérica } g / d l \\
n=38\end{array}$} & Mayor de 10 & $44.7(17)$ \\
\hline & Entre $8.5-10$ & $15.7(6)$ \\
\hline & Menor de 8.5 & $39.4(15)$ \\
\hline \multirow{3}{*}{ Calcio sérico $\mathrm{mg} / \mathrm{dl} \mathrm{n}=35$} & Entre $8.8-10.3$ & $60(21)$ \\
\hline & Entre $10.4-12.5$ & $22.8(8)$ \\
\hline & Mayor de 12.5 & $17.1(6)$ \\
\hline \multirow{2}{*}{ Creatinina sérica $\mathrm{mg} / \mathrm{dl} n=38$} & Menor de 2 & $73.6(28)$ \\
\hline & Mayor o igual a 2 & $26.3(10)$ \\
\hline \multirow{2}{*}{ Albumina sérica $g / d l n=31$} & Menor de 3.5 & $67.7(21)$ \\
\hline & Mayor o igual a 3.5 & $32.2(10)$ \\
\hline \multirow{3}{*}{$\begin{array}{l}\beta_{2} \text { microglobulina sérica } \\
\mathrm{mg} / / \mathrm{n}=29\end{array}$} & Menor de 3.5 & $44.8(13)$ \\
\hline & Entre $3.5-5.4$ & $27.5(8)$ \\
\hline & Mayor o igual a 5.5 & $27.5(8)$ \\
\hline \multirow{3}{*}{ Lesiones líticas $n=30$} & 0 a I lesiones & $17.9(7)$ \\
\hline & 2 a 3 lesiones & $12.8(5)$ \\
\hline & Más de 3 lesiones & $46.1(18)$ \\
\hline
\end{tabular}

Tabla 2. Estadificación según Durie-Salmon e International Staging System (ISS)

\begin{tabular}{|c|c|}
\hline \multicolumn{2}{|c|}{ Durie-Salmon } \\
\hline I & $5.1(2)$ \\
II & $5.1(2)$ \\
III & $89.7(35)$ \\
A & $51.2(20)$ \\
B & $48.7(19)$ \\
\hline
\end{tabular}

\begin{tabular}{|c|c|}
\hline \multicolumn{2}{|c|}{ International Staging System (ISS) } \\
\hline Estadio, =29 & $\%(\mathrm{n})$ \\
\hline 1 & $31(9)$ \\
2 & $13.7(4)$ \\
3 & $55.1(16)$ \\
\hline
\end{tabular}


el reporte $(n=25 / 32)$, de los cuales $84 \%(n=21 / 25)$ reveló pico monoclonal gamma y en el $16 \%$ restante $(n=4 / 25)$ fue beta.

- De las inmunoglobulinas séricas la IgG y la IgA se encontraron elevadas en una proporción igual de pacientes, 33,3\% ( $n=11 / 33)$, mientras que la IgM solo se halló alta en 3,2\% de los evaluados $(n=1 / 31)$.

- Las cadenas livianas séricas fueron reportadas solo en 15 de los 39 casos observando elevación del subtipo kappa en $46,6 \%(n=7)$ y lambda en $33,3 \%$ $(n=5)$; por su parte las cadenas livianas urinarias solo fueron reportadas en 13 de los 39 , con valores altos del componente kappa en $100 \%$ de estos y del lambda el 76,9\% $(n=10)$.

- La inmunofijación sérica se reportó solo en siete de 39 pacientes siendo positiva en 85,7\% $(n=6 / 7)$ para el subtipo IgG-kappa y solo en $14,2 \%(n=1 / 7)$ para el subtipo IgA-lambda. De igual manera la inmunofijación urinaria solo fue reportada en ocho de los 39 siendo positiva en $75 \%(n=6 / 8)$ para el subtipo IgG-kappa y $12,5 \%(n=1 / 8)$ IgA-lambda.

Al $12,8 \%(n=5 / 39)$ se les realizaron estudios citogenéticos de los cuales fueron cariotipo en cuatro pacientes y FISH a uno, sin hallar alteraciones. Solo se encontró evaluación objetiva de la escala funcional según ECOG en el $41 \%$ de los casos $(n=16 / 39)$, con reporte de $24,9 \%$ con ECOG mayor de 1 .

Los esquemas de quimioterapia utilizados, frecuencia y tipo de respuesta alcanzada durante la primera línea de tratamiento se representan en la Tabla 3. En esta evidenciamos que los más comunes fueron talidomida-dexametasona en el $23 \%$ y la basada en bortezomib con un porcentaje igual, usada en especial en los dos últimos años del estudio poniendo de manifiesto la tendencia a la utilización de este medicamento en primera línea de tratamiento de acuerdo con las recomendaciones internacionales actuales. El $86 \%$ de los pacientes obtuvo por lo menos una muy buena respuesta parcial.

Al reportar los resultados de los estudios de inmunohistoquímica y citometría de flujo no se expresan las características inmunofenotípicas de las células neoplásicas sino el porcentaje de infiltración de estas. El $56,4 \%$ de los pacientes está vivo a la fecha de análisis de datos del presente estudio $(n=22 / 39)$; el 33,3\% ya falleció $(n=14 / 39)$ y no se pudo obtener información en el 7,6\% de los casos $(n=3 / 39)$.

\begin{tabular}{|c|c|c|c|c|c|c|c|c|}
\hline \multicolumn{9}{|c|}{ Esquemas de quimioterapia* } \\
\hline Años de uso & 2003-2005 & 2006-2009 & $2006-2010$ & 2006-2009 & 2003 & $2009-2010$ & $2009-2010$ & \\
\hline Respuesta completa & 1 & & & I & & - & & 2 \\
\hline Respuesta parcial & & $=$ & 1 & 1 & & 1 & 1 & 4 \\
\hline Muy buena respuesta parcial & 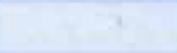 & & 4 & 3 & & 2 & & 9 \\
\hline Refractariedad & 1 & & & & & & & 1 \\
\hline
\end{tabular}

* Se administró quimioterapia a 39 pacientes y no se obtuvo información de la respuesta obtenida en 17 (VAD: 5); (MP:3); (MPT:1); (TD:2); (VD:2); (CP:3); (VTD:1). VAD: vincristina - adriamicina-dexametasona; MP: melfalan - prednisona; MPT: melfalan - prednisona-talidomida; TD: talidomida - dexametasona; CP: ciclofosfamida - prednisona; VD: bortezomib - dexametasona y VID: bortezomib-talidomida-dexametasona. 


\section{Discusión}

La literatura internacional reporta que las tres manifestaciones clínicas más encontradas en mieloma múltiple son anemia (73\%), dolores óseos (58\%) y enfermedad renal crónica (48\%). ${ }^{4}$ En nuestra serie fueron dolores óseos $(71,7 \%)$, síntomas B $(61,5 \%)$ y manifestaciones generales (astenia, adinamia y malestar) 43,5\%; cerca de la mitad son menores de 60 años $(53,8 \%)$ y resalta el 20,5\% inferior de 50 diferente del $10 \%$ según los reportes internacionales. ${ }^{4,18}$ También, en contraste con los informes de la literatura ${ }^{4,8}$ en esta serie la frecuencia de IgA e IgM como componente $\mathbf{M}$ fue similar. Solo el $12,8 \%$ de nuestros casos se les hicieron estudios citogenéticos, situación que dificulta la adecuada estratificación del riesgo de la gran mayoría de ellos, limitando el direccionamiento terapéutico adecuado de manera temprana sobre todo en aquellos sin contraindicaciones para trasplante de precursores hematopoyéticos.

El primer estudio citogenético en nuestra serie fue realizado en 2006, evidenciando que desde que se cuenta con la posibilidad de realizar este tipo de estudios en el país, nuestro servicio se ha acogido a las recomendaciones internacionales en la estratificación de riesgo de los pacientes con mieloma múltiple ${ }^{19}$, por lo que el reducido porcentaje de pacientes en los cuales se reportó la citogenética pone de manifiesto las limitaciones inherentes al funcionamiento del sistema de seguridad social en el país.

El factor pronóstico evaluado en la mayoría de los pacientes fue el ISS identificándose un $69 \%$ en estadios 2 ó 3 y de estos un $63 \%$ obtuvo por lo menos una muy buena respuesta parcial posterior a la quimioterapia de primera línea. De los 39 casos, en 30 se definió, desde la evaluación inicial si eran o no candidatos a trasplante, de ellos el 70\% (21/30) estaba en la primera categoría; estos fueron remitidos a centros de trasplante. De ellos, cinco no lo fueron por falla terapéutica (cuatro por progresión y uno por refractariedad). Solo cuatro de los 16 restantes continuó controles en este servicio y por lo tanto se pudo confirmar que fueron trasplantados como consolidación de primera línea de tratamiento. Después de una búsqueda de información en centros especializados de la ciudad, se logró confirmar la realización en otros tres para un total de siete. Va más allá de los objetivos de este trabajo describir los desenlaces y la evolución después del trasplante.

El tratamiento del mieloma múltiple ha evolucionado en forma dramática en las últimas décadas, pasando por combinaciones de melfalan-prednisona (1960) en los no candidatos a trasplante o ancianos, quimioterapia basada en vincristina, adriamicina y dexametasona (VAD) sola o asociada con trasplante (1980), hasta la asociación de quimioterapia con medicamentos novedosos combinada con trasplante autólogo de células madres hematopoyéticas (2000), con mejorías sucesivas en las tasas de respuesta y de supervivencia global, favoreciendo la administración de quimioterapia con medicamentos novedosos asociada con trasplante..$^{19,20}$ Así, este último esquema (quimioterapia + trasplante) es considerado hoy en día como el tratamiento estándar de primera línea en todos los pacientes con diagnóstico reciente de mieloma múltiple sintomático, sin contraindicaciones para tal procedimiento..$^{9,14,16}$

Las tasas de respuesta global esperadas para quimioterapia VAD eran alrededor de $55 \%^{17}$, pero su administración condicionaba algunos inconvenientes que incluían la necesidad de hospitalización para infusión de medicamentos durante 24 horas por cuatro días, neurotoxicidad por vincristina, alopecia por adriamicina y el riesgo de complicaciones infecciosas, situaciones estas que conllevaron a la búsqueda de nuevos opciones.

A comienzos de la década del 2000, la talidomida muestra resultados prometedores en el manejo del mieloma múltiple en recaída o refractario ${ }^{17,21,22}$, siendo después utilizada por varios grupos como indicación de primera línea, combinada con quimioterapia convencional o dexametasona, encontrándose una tasa de respuesta global de $76 \%$ cuando se combina con dexametasona. ${ }^{23,24}$ Más adelante se introdujeron otros agentes novedosos al arsenal terapéutico del mieloma múltiple que incluyen bortezomib y lenalidomida, utilizados en combinación con esteroides, mostrando mejoría significativa en la tasa de respuesta (mayor al 80\%), así como una mayor supervivencia libre de 
evento y global; estos medicamentos hacen parte de las opciones ofrecidas hoy como primera línea. ${ }^{19}$

Aquellos con diagnóstico de mieloma múltiple sintomático son candidatos a intervención basada en quimioterapia sola o seguida de trasplante autólogo de células madres hematopoyéticas; la escogencia del tratamiento inicial depende de dos factores principales: de ser o no candidato a trasplante y de la estratificación de riesgo (ISS y citogenética). Los candidatos a trasplante si son de alto riesgo reciben alrededor de cuatro ciclos de quimioterapia de alta intensidad, con el objetivo de lograr respuesta completa y son consolidados con trasplante; mientras que los considerados como riesgo estándar, podrían recibir quimioterapia de alta intensidad y si alcanzan una respuesta completa o completa estricta pueden mantenerse en observación, reservando el trasplante para la primera recaída. ${ }^{9}$

Las guías actuales del mieloma múltiple sintomático recomiendan quimioterapia basada en bortezomib como tratamiento de primera línea para pacientes

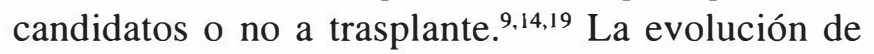
esta terapia en nuestra serie, refleja los cambios de tendencia en el mundo. El protocolo VAD fue la base del manejo de inducción hasta 2006, cuando se empezaron a utilizar los protocolos basados en talidomida. Después, entre 2008 y 2009, se introduce bortezomib como tratamiento de primera línea.

La tasa de respuesta global a talidomida-dexametasona fue de $85,7 \%$, a melfalan-prednisona-talidomida de $83 \%$ y a quimioterapia basada en bortezomib del 100\% (Tabla 3). Aunque estos resultados no son comparables con las publicaciones internacionales por el reducido número de pacientes, sí reflejan la tendencia en las tasas de respuesta global de cada uno de estos esquemas.

Los datos reportados revelan que en Bogotá y en nuestra institución contamos con herramientas adecuadas para el diagnóstico, estadificación y pronóstico del mieloma múltiple. Por otro lado, debido a limitaciones derivadas de los diferentes procesos administrativos que conllevan su utilización, están siendo subutilizados repercutiendo en la adecuada estratificación de riesgo para direccionar de la mejor forma su tratamiento.

\section{Referencias}

1. Ries LAG, Eisner MP, Kosary C, et al. SEER Cancer Statistics Review, 19752001. Bethesda, MD, National Cancer Institute. 2004.

2. Blade J, Kyle RA. Multiple myeloma in young patients: clinical presentation and treatment approach. Leuk Lymphoma. 1998 Aug;30(5-6):493-50I.

3. Jemal A, Siegel R, Ward E, et al. Cancer statistics, 2009. CA Cancer J Clin. 2009;59:225.

4. Kyle RA, Gertz M, Witzig TE, et al. Review of 1027 patients with newly diagnosed multiple myeloma. Mayo Clin Proc. 2003;78:21.

5. Ferlay J, Shin H. Bray F, Forman D, Mathers C PD. Estimates of worldwide burden of cancer in 2008: GLOBOCAN 2008. Int J Clin Pract. 2010;127:2893-917.

6. Instituto Nacional de Cancerología. Anuario Estadístico. 2009;7.

7. Smith A, Wisloff $F$, Samson D. Guidelines on the diagnosis and management of multiple myeloma 2005. Br J Haematol. 2006;132:410.

8. Rajkumar SV, Dispenzieri A, Kyle RA. Monoclonal gammopathy of undertermined significance, Waldenstrom macroglobulinemia, AL amyloidosis, and related plasma cell disorders: diagnosis and treatment. Mayo Clin Proc. 2006;81:693.

9. Kumar SK, Mikhael JR, Buadi FK, Dingli D, Dispenzieri A, Fonseca R, et al. Management of newly diagnosed symptomatic multiple myeloma: updated Mayo Stratification of Myeloma and Risk-Adapted Therapy (mSMART) consensus guidelines. Mayo Clin Proc. 2009 Dec;84(12):1095-110.

10. Greipp PR, San MJ, Durie BG, et al. International staging system for multiple myeloma. J Clin Oncol. 2005;23:3412.

11. Fonseca R, Blood E, Rue M, et al. Clinical and biologic implications of recurrent genomic aberrations in myeloma. Blood. 2003;101:4569.

12. Durie BG, Harousseau JL, Miguel JS, et al. International uniform response criteria for multiple myeloma. Leukemia. 2006;20:1467.

13. Munshi NC. Plasma cell disorders: an historical perspective. Hematology 2008;2008(297).

14. Anderson KC, Faber E, Somlo G, Alsina M, Huff CA, Stockerl-Goldstein KE, et al. NCCN Clinical Practice Guidelines in Oncology. National Comprehensive Cancer Network. 2011;1.2011:1-53.

15. Attal M, Harousseau JL, Stoppa AM, Sotto J, Fuzibet J, Rossi JF, et al. A prospective, randomized trial of autologous bone marrow transplantation and chemotherapy in multiple myeloma. Intergroupe Français du Myélome. N Engl J Med. 1996;335(2):91-7.

16. Rajkumar SV. Treatment of multiple myeloma. Nat Rev Clin Oncol. 2011;8(8):479-81.

17. Palumbo A, Triolo S, Argentino C, Bringhen S, Dominietto A, Rus C, et al. Dose-intensive melphalan with stem cell support (MEL100) is superior to standard treatment in elderly myeloma patients. Blood. 1999;94(4):1248-53.

18. Kyle RA, Themeau TM, Rajkumar SV, et al. Incidence of multiple myeloma in Olmsted County, Minnesota: Trend over 6 decades. Cancer. 2004;101:2667.

19. Kapoor P, Rajkumar SV. Update on risk stratification and treatment of newly diagnosed multiple myeloma. Int J Hematol. 2011;10:1007.

20. Barlogie B, Alexanian R, Dicke KA, et al. High-dose chemoradiotherapy and autologous bone marrow transplantation for resistant multiple myeloma. $\mathrm{Br} \mathrm{J}$ Haematol. 1987;66(1):55-62.

21. Alexanian R, Weber D, Giralt S, Delasalle K, Smith T, et al. Impact of complete remission with intensive therapy in patients with responsive multiple myeloma. Bone Marrow Transplant. 2001;27(10):1037-43.

22. Singhal S, Mehta J, Desikan R, et al. Antitumor activity of thalidomide in refractory multiple myeloma. N Engl J Med. 1999;341:1565-71.

23. Cavo M, Zamagni E, Tosi P, Taccetti P, Cellini C, Canning C. Superiority of thalidomide and dexamethasone over vincristine - doxorubicindexamethasone (VAD) as primary therapy in preparation for autologous transplantation for multiple myeloma. Blood. 2005; 106:35-9.

24. Rajkumar SV. Multiple myeloma: the death of VAD as initial therapy. Blood 2005;106:2-3. 